\title{
THE DOMAIN SPACE OF AN ANALYTIC COMPOSITION OPERATOR
}

\author{
THOMAS DOMENIG, HANS JARCHOW and REINHARD RIEDL
}

(Received 20 April 1998; revised 10 September 1998)

Communicated by P. G. Dodds

\begin{abstract}
In this paper we show that, for analytic composition operators between weighted Bergman spaces (including Hardy spaces) and as far as boundedness, compactness, order boundedness and certain summing properties of the adjoint are concerned, it is possible to modify domain spaces in a systematic fashion: there is a space of analytic functions which embeds continuously into each of the spaces under consideration and on which the above properties of the operator are decided.

A remarkable consequence is that, in the setting of composition operators between weighted Bergman spaces, the properties in question can be identified as properties of the operator as a map between appropriately chosen Hilbert spaces.
\end{abstract}

1991 Mathematics subject classification (Amer. Math. Soc.): primary 47B38; secondary 30D55, 46E15. Keywords and phrases: Composition operators, weighted Bergman spaces, Hardy spaces, boundedness, compactness, absolutely summing operators.

\section{Preparations}

Let $D$ be the open unit disk $\{z \in \mathbb{C}:|z|<1\}$ in the complex plane. Normalized area measure on $D$ will be denoted by $\sigma$, so $d \sigma(z)=\pi^{-1} d x d y$. For each $\alpha>-1$, a probability measure $\sigma_{\alpha}$ on $D$ is given by $d \sigma_{\alpha}(z):=(\alpha+1)\left(1-|z|^{2}\right)^{\alpha} d \sigma(z)$. For $0<p<\infty$, we denote the canonical ( $p$-) norm on the corresponding Lebesgue space $L^{p}\left(\sigma_{\alpha}\right)$ by $\|\cdot\|_{p, \alpha}$.

The analytic functions $f: D \rightarrow \mathbb{C}$ such that $\|f\|_{\alpha, p}<\infty$ form a closed subspace of $L^{p}\left(\sigma_{\alpha}\right)$ which we denote by $\mathscr{A}_{\alpha}^{p}$. This is the weighted Bergman space defined by $\alpha$ and $p$; it is a Banach space if $1 \leq p<\infty$ and a $p$-Banach space when $0<p<1$. The polynomials form a dense subspace in $\mathscr{A}_{\alpha}^{p}$.

The second author was supported by the Swiss National Funds.

(C) 1999 Australian Mathematical Society 0263-6115/99 \$A2.00+0.00 
We write $\mathbb{J}:=\{z \in \mathbb{C}:|z|=1\}$ for the boundary of $D$ and $m$ for normalized Lebesgue measure on $\mathbb{T}$; so $d m\left(e^{i t}\right)=d t / 2 \pi$. The Hardy space $H^{p}$ $(0<p<\infty)$ consists of all analytic functions $f$ on $D$ such that $\|f\|_{p}:=$ $\sup _{r<1}\left(\int_{-\pi}^{\pi}\left|f\left(r e^{i t}\right)\right|^{p} \frac{d t}{2 \pi}\right)^{1 / p}<\infty$. Again, $\left[H^{p},\|\cdot\|_{p}\right]$ is a Banach space when $1 \leq p<\infty$, and a $p$-Banach space when $0<p<1$.

As usual, $H^{\infty}$ is the Banach space of all bounded analytic functions on $D$; its norm is $\|f\|_{\infty}=\sup _{z \in D}|f(z)|$.

By Fatou's Theorem, given $p$ and $f \in H^{p}, \lim _{r / 1} f\left(r e^{i t}\right)$ exists for (Lebesgue) almost all $t$. The resulting 'function' $\tilde{f}: \mathbb{T} \rightarrow \mathbb{C}$ belongs to $L^{p}(m)$, and $H^{p} \rightarrow$ $L^{p}(m): f \mapsto \widetilde{f}$ is an isometric isomorphism onto the closure (weak*-closure if $p=\infty$ ) of all polynomials in $L^{p}(m)$. Accordingly, we may identify $H^{p}$ with a closed subspace of $L^{p}(m)$, and for $f \in H^{p}$ we shall simply write $f$ instead of $\tilde{f}$. See [6] for details. It is easy to see that if $-1<\alpha<\beta$, then $H^{p} \hookrightarrow \mathscr{A}_{\alpha}^{p} \hookrightarrow \mathscr{A}_{\beta}^{p}$ with continuous inclusions for all values of $p$.

\section{The spaces $\mathscr{A}_{\alpha}^{(p)}$}

For each $z \in D$, the function $w \mapsto k_{z}(w):=(1-\bar{z} w)^{-1}$ belongs to $H^{\infty}$. The map $(z, w) \mapsto k_{z}(w)$ is the reproducing kernel for the Hilbert space $H^{2}$ : we have $f(z)=\left(f \mid k_{z}\right)$ for all $f \in H^{2}$ and $z \in D$. We use $(\cdot \cdot)$ to denote the scalar product of a Hilbert space.

Each of the Hilbert spaces $\mathscr{A}_{\alpha}^{2}, \alpha>-1$, has a reproducing kernel $(z, w) \mapsto k_{z}^{(\alpha)}(w)$; it is well known [19, p. 120] that $k_{z}^{(\alpha)}(w):=k_{z}(w)^{2+\alpha}(z, w \in D)$. We shall therefore also write $\mathscr{A}_{-1}^{p}$ instead of $H^{p}(0<p<\infty)$, and $\sigma_{-1}$ instead of $m$.

Let $\alpha \geq-1$. The norm of $k_{z}^{(\alpha)}$ in the Hilbert space $\mathscr{A}_{\alpha}^{2}$ is $\left\|k_{z}^{(\alpha)}\right\|_{\mathscr{S}_{\alpha}^{2}}=\left(1-|z|^{2}\right)^{-(2+\alpha) / 2}$ $[19$, p. 54$]$, and so the functions

$$
\mu_{\alpha, p, z}(w):=\left(\frac{1-|z|^{2}}{(1-\bar{z} w)^{2}}\right)^{(2+\alpha) / p} \quad(w \in D)
$$

are norm one functions in $\mathscr{A}_{\alpha}^{p}(0<p<\infty)$. Let $\mathscr{A}_{\alpha}^{(p)}$ be the space of all $f \in \mathscr{A}_{\alpha}^{p}$ for which numbers $a_{n} \in \mathbb{C}$ and $z_{n} \in D$ exist such that $\sum_{n}\left|a_{n}\right|<\infty$ and

$$
f(w)=\sum_{n=1}^{\infty} a_{n} \mu_{\alpha, p, z_{n}}(w) \quad \forall w \in D .
$$

$\mathscr{A}_{\alpha}^{(p)}$ is a linear space on which

$$
\|f\|_{\mathcal{S}_{\alpha}^{(p)}}:=\inf \left\{\sum_{n=1}^{\infty}\left|a_{n}\right|:(*) \text { holds }\right\}
$$


is a norm. We obtain a Banach space in this way: $\ell^{1}(D) \rightarrow \mathscr{A}_{\alpha}^{(p)}:\left(a_{z}\right)_{z \in D} \mapsto$ $\sum_{z \in D} a_{z} \mu_{\alpha, p, z}$ is a metric surjection. It is also clear that if $p \geq 1$, then $\mathscr{A}_{\alpha}^{(p)}$ embeds contractively into $\mathscr{A}_{\alpha}^{p}$ and that, thanks to the reproducing nature of the $\mu_{\alpha, p, z}$ 's, this embedding has dense range.

Moreover, two further consequences of the definition will be important for us.

PROPOSITION 1. (a) If $p, p^{\prime}>0$ and $\alpha, \alpha^{\prime} \geq-1$ are such that $(2+\alpha) / p=$ $\left(2+\alpha^{\prime}\right) / p^{\prime}$, then $\mathscr{A}_{\alpha}^{(p)}$ and $\mathscr{A}_{\alpha^{\prime}}^{\left(p^{\prime}\right)}$ coincide as Banach spaces.

(b) If $\alpha>-1$, then $\mathscr{A}_{\alpha}^{1}=\mathscr{A}_{\alpha}^{(1)}$, with equivalence of norms.

PROOF. (a) is immediate from the definitions, and (b) is due to the fact that atomic decomposition is available in weighted Bergman spaces: just apply [11, Proposition 4.5] with $N=1$ and $\eta=2+\alpha$.

In particular, if $2+\alpha>p$, then $\mathscr{A}_{\alpha}^{(p)}$ is isomorphic to $\ell^{1}$ (since $\mathscr{A}_{\alpha^{\prime}}^{1}$ has this property when $\alpha^{\prime}>-1$ ).

We shall use several times the following result given by Shapiro [14]: if $0<p<1$ then the Banach space envelope of $\mathscr{A}_{\alpha}^{p}$ is $\mathscr{A}_{\tau}^{1}$, where $\tau:=(2+\alpha) / p-2$. Note that $\mathscr{A}_{\alpha}^{(p)}=\mathscr{A}_{\tau}^{(1)}=\mathscr{A}_{\tau}^{1}$ (isomorphically) in this case.

\section{Boundedness and compactness}

We denote by $\Phi$ the set of all analytic functions $\varphi: D \rightarrow D$. Given $\varphi \in \Phi$, $f \mapsto f \circ \varphi$ defines a bounded linear operator between suitable spaces of analytic functions on $D$; if this is the case, we denote this operator by $C_{\varphi}$ and refer to the composition operator induced by $\varphi$.

We start by looking at boundedness on weighted Bergman spaces:

PROPOSITION 2. Let $\varphi \in \Phi, \alpha, \beta \geq-1$ and $q \geq p>0$ with $q \geq 1$ be given. Then the following statements are equivalent:

(i) $C_{\varphi}$ exists as a bounded operator $\mathscr{A}_{\alpha}^{p} \rightarrow \mathscr{A}_{\beta}^{q}$,

(ii) $C_{\varphi}$ exists as a bounded operator $\mathscr{A}_{\alpha}^{(p)} \rightarrow \mathscr{A}_{\beta}^{q}$,

(iii) $\sup _{z \in D}\left\|C_{\varphi}\left(\mu_{\alpha, p, z}\right)\right\|_{\mathscr{A}_{\beta}^{q}}<\infty$.

PROOF. (i) $\Rightarrow$ (ii) follows easily by restriction whenever $\mathscr{A}_{\alpha}^{p}$ is a Banach space. Otherwise we can rely on a 'trick of multiplying exponents': if $C_{\varphi}$ is bounded as an operator $\mathscr{A}_{\alpha}^{p} \rightarrow \mathscr{A}_{\beta}^{q}$, then it is also bounded as an operator $\mathscr{A}_{\alpha}^{s p} \rightarrow \mathscr{A}_{\beta}^{s q}$, for any $s>0$. This follows from [18, Corollary 4.4]; see [7] for the case $\alpha=-1$. Choosing $s \geq 1$ so that $s p \geq 1$, we get that $C_{\varphi}$ is bounded as an operator $\mathscr{A}_{\alpha}^{(s p)} \rightarrow \mathscr{A}_{\beta}^{s q}$. A 
quick perusal of the definition leads from here to boundedness of $C_{\varphi}$ as an operator $\mathscr{A}_{\alpha}^{(p)} \rightarrow \mathscr{A}_{\beta}^{q}$.

(ii) $\Leftrightarrow$ (iii) is obvious. To prove that (i) follows from this, we apply the same strategy. If $C_{\varphi}$ is bounded as an operator $\mathscr{A}_{\alpha}^{(p)} \rightarrow \mathscr{A}_{\beta}^{q}$, then it is bounded from $\mathscr{A}_{\alpha}^{(1)}=\mathscr{A}_{\alpha}^{1}$ to $\mathscr{A}_{\beta}^{q / p}$. As before, boundedness of $C_{\varphi}: \mathscr{A}_{\alpha}^{p} \rightarrow \mathscr{A}_{\beta}^{q}$ follows suit.

As is to be expected, compactness in the situation of the above proposition only requires passing to the corresponding 'little o'-condition.

Proposition 3. Let $\varphi \in \Phi, \alpha, \beta \geq-1$ and $q \geq p>0$ with $q \geq 1$ be given. The following statements are equivalent:

(i) $C_{\varphi}$ exists as a compact operator $\mathscr{A}_{\alpha}^{p} \rightarrow \mathscr{A}_{\beta}^{q}$,

(ii) $C_{\varphi}$ exists as a compact operator $\mathscr{A}_{\alpha}^{(p)} \rightarrow \mathscr{A}_{\beta}^{q}$,

(iii) $\lim _{|z| / 1}\left\|C_{\varphi}\left(\mu_{\alpha, p, z}\right)\right\|_{\alpha_{\beta}^{q}}=0$.

PROOF. (i) $\Rightarrow$ (ii) is proved as before, using [18, Theorem 5.1].

(ii) $\Rightarrow$ (iii) comes from the fact that $\lim _{|z| \not \mid} \mu_{\alpha, p, z}=0$ holds pointwise on $D$. To prove (iii) $\Rightarrow$ (ii), consider the canonical surjection $Q: \ell^{1}(D) \rightarrow \mathscr{A}_{\alpha}^{(p)}$ which maps the standard unit vectors $e_{z}$ of $\ell^{1}(D)$ to $\mu_{\alpha, p . z}$, and use that relative compactness of $\left\{C_{\varphi}\left(Q e_{z}\right): z \in D\right\}$ is equivalent to compactness of $C_{\varphi} \circ Q$, and hence of $C_{\varphi}$; compare [2, p. 114].

(ii) $\Rightarrow$ (i) can be obtained as before.

REMARKS. (a) The hypothesis $q \geq p \vee 1$ was needed to ensure that our range spaces are always Banach spaces.

(b) Rather than referring to [18] in the preceding propositions, we could also have applied a modified Carleson measure type argument which was used in [13] in a Hardy space situation. We provide a sketch of (iii) $\Rightarrow$ (ii) for the case of compact operators: We define $\sigma_{\beta, \varphi}(\cdot):=\sigma_{\beta}\left(\varphi^{-1}(\cdot)\right)$ on Borel sets. Given $\varepsilon>0$, we can find $1 / 2<\delta<1$ such that

$$
\int_{D}\left(\frac{1-|z|^{2}}{1-\left.\bar{z} w\right|^{2}}\right)^{(2+\alpha) q / p} d \sigma_{\beta, \varphi}(w)<\varepsilon \quad \text { for } \delta<|z|<1 .
$$

We fix $0<r<2(1-\delta)$ and $0<\theta \leq 2 \pi$, set $z_{0}:=\left(1-\frac{r}{2}\right) e^{i \theta}$ and consider the Euclidean disk $B$ with center $z_{0}$ and radius $r / 2$. Since $1-\left|z_{0}\right| \leq\left|1-\overline{z_{0}} w\right|$ for all $w \in D$, we can find a constant $C=C(\alpha, p, q)$ such that

$$
\begin{aligned}
\sigma_{\beta, \varphi}(B) & \leq C\left(1-\left|z_{0}\right|^{2}\right)^{(2+\alpha) q / p} \int_{B}\left(\frac{1-\left|z_{0}\right|^{2}}{\left|1-\overline{z_{0}} w\right|^{2}}\right)^{(2+\alpha) q / p} d \sigma_{\beta, \varphi}(w) \\
& \leq C\left(1-\left|z_{0}\right|^{2}\right)^{(2+\alpha) q / p} \varepsilon \leq C r^{(2+\alpha) q / p} \varepsilon .
\end{aligned}
$$


It follows that $\mathscr{A}_{\alpha}^{p}$ embeds compactly into $L^{q}\left(\sigma_{\beta, \varphi}\right)$, and this is just another way of stating that $C_{\varphi}$ exists as a compact operator from $\mathscr{A}_{\alpha}^{p}$ into $\mathscr{A}_{\beta}^{q}$.

A version for Carleson measures in the case of Hardy spaces and for $p=q$ can be found in Zhu [19, pp. 171].

(c) For $p \leq 1$, the preceding results can also be proved using that for $\tau=(2+$ $\alpha) / p-2, \mathscr{A}_{\tau}^{1}$ is the Banach space envelope of $\mathscr{A}_{\alpha}^{p}$ ([14]).

As a corollary, we may state:

COROllary 4. Let $\varphi \in \Phi, 0<p, p^{\prime}, q, q^{\prime}<\infty$ be such that $1 \vee p \leq q$ and $1 \vee p^{\prime} \leq q^{\prime}$, and $\alpha, \alpha^{\prime}, \beta \geq-1$ be given. If $(2+\alpha) p^{\prime} q=\left(2+\alpha^{\prime}\right) p q^{\prime}$, then $C_{\varphi}$ maps $\mathscr{A}_{\alpha}^{p}$ boundedly (respectively compactly) into $\mathscr{A}_{\beta}^{q}$ if and only if it has the same property as an operator from $\mathscr{A}_{\alpha^{\prime}}^{p^{\prime}}$ to $\mathscr{A}_{\beta}^{q^{\prime}}$.

PROOF. This is clear if $q=q^{\prime}$. The general case is obtained using once more the 'trick of multiplying exponents'.

This result fails for example for weakly compact composition operators. For a specific example, take $\alpha=0, \alpha^{\prime}=\beta=-1, p^{\prime}=1 / 2, q^{\prime}=1$ and $p=q>1$. Then $(2+\alpha) p^{\prime} q=\left(2+\alpha^{\prime}\right) p q^{\prime}$ so that we can compare $C_{\varphi}: \mathscr{A}_{0}^{p} \rightarrow H^{p}$ and $C_{\varphi}: H^{1 / 2} \rightarrow H^{1}$. It was shown in [5], via the existence of suitable non-compact diagonal operators $\ell^{p} \rightarrow \ell^{p}$, that there exist operators $C_{\varphi}: \mathscr{A}_{0}^{p} \rightarrow H^{p}$ which are not compact; but since $p>1$, this operator is weakly compact. On the other hand, as was shown in [9], weak compactness of $C_{\varphi}: H^{1 / 2} \rightarrow H^{1}$ always entails compactness. (The weak topology of $H^{1 / 2}$ is taken with respect to the separating dual of this space.)

On the other side we may now state that, within our setting, boundedness and compactness of composition operators can entirely be phrased in terms of Hilbert space operators:

Corollary 5. Let $\varphi \in \Phi, \alpha, \beta \geq-1$ and $0<p, q<\infty$ be such that $1 \vee p \leq q$. Set $\alpha^{\prime}=(2+\alpha)(q / p)-2$. Then $C_{\varphi}$ exists as a bounded (compact) operator $\mathscr{A}_{\alpha}^{p} \rightarrow \mathscr{A}_{\beta}^{q}$ if and only if it exists as a bounded (compact) operator $\mathscr{A}_{\alpha^{\prime}}^{2} \rightarrow \mathscr{A}_{\beta}^{2}$.

\section{Order boundedness}

Let $X$ be a quasi-Banach space and $Y$ a subspace of a quasi-Banach lattice $L$. We say that an operator $u: X \rightarrow Y$ is order bounded if it maps the unit ball $B_{X}$ of $X$ into an order interval of $L$ : for some $h \geq 0$ in $L$ we have $|u x| \leq h$ for all $x \in B_{X}$. Within the setting of composition operators, the concept has been considered in [7] and [4] for Hardy spaces, and in [5] for weighted Bergman spaces.

Propositions 2 and 3 can be carried over to order bounded composition operators. 
Proposition 6. Given $\varphi \in \Phi, \alpha, \beta \geq-1, p>0$ and $q \geq 1$, the following are equivalent:

(i) $C_{\varphi}$ exists as an order bounded operator $\mathscr{A}_{\alpha}^{p} \rightarrow \mathscr{A}_{\beta}^{q}$,

(ii) $C_{\varphi}$ exists as an order bounded operator $\mathscr{A}_{\alpha}^{(p)} \rightarrow \mathscr{A}_{\beta}^{q}$,

(iii) $\left(1-|\varphi|^{2}\right)^{-1}$ belongs to $L^{(2+\alpha) q / p}\left(d \sigma_{\beta}\right)$.

Note: (iii) reveals that, for fixed $\alpha$ and $\beta$, order boundedness also depends only on the ratio $q / p$.

PROOF. (i) $\Rightarrow$ (ii): Suppose that $C_{\varphi}$ exists as an order bounded operator $\mathscr{A}_{\alpha}^{p} \rightarrow \mathscr{A}_{\beta}^{q}$. If $p \geq 1$, then restriction yields $C_{\varphi}$ as an order bounded operator from $\mathscr{A}_{\alpha}^{(p)}$ to $\mathscr{A}_{\beta}^{q}$. If $p<1$, we apply the results from [14] together with a straightforward convexity argument to conclude that $C_{\varphi}$ maps $\mathscr{A}_{\tau}^{1}$ order boundedly into $\mathscr{A}_{\beta}^{q}$, where $\tau=(2+\alpha) / p-2$. But $\mathscr{A}_{\alpha}^{(p)}=\mathscr{A}_{\tau}^{(1)}=\mathscr{A}_{\tau}^{1}$ since $2+\alpha=p(2+\tau)$.

(ii) $\Rightarrow$ (iii): This is immediate since $\sup _{z \in D}\left(1-|z|^{2}\right)|1-\bar{z} \varphi(w)|^{-2}=\left(1-|\varphi(w)|^{2}\right)^{-1}$ for each $w \in D$.

(iii) $\Rightarrow$ (i): We show that there is a constant $c$ (depending on $\alpha$ and $p$ ) such that

$$
\left|C_{\varphi} f(z)\right| \leq c\left(\frac{1}{1-|\varphi(z)|^{2}}\right)^{(2+\alpha) / p}
$$

for all $f \in B_{\mathscr{\alpha}_{\alpha}^{p}}$ and $z \in D$. To this end, we need to estimate the norm of the reproducing kernel $k_{z}^{(\alpha)}$ as an element of $\left(\mathscr{A}_{\alpha}^{p}\right)^{*}$. If $p \geq 1$, then an appeal to [19, Lemma 4.2.2] provides us with a constant $c(\alpha, p)$ such that

$$
\left|C_{\varphi}(z)\right|=|f(\varphi(z))| \leq\left\|k_{\varphi(z)}^{(\alpha)}\right\|_{\left(\mathcal{A d}_{\alpha}^{p}\right)^{*}} \leq c(\alpha, p)\left(\frac{1}{1-|\varphi(z)|^{2}}\right)^{(2+\alpha) / p} .
$$

If $0<p<1$, then we set again $\tau=(2+\alpha) / p-2$ and use [14] to get $\left(\mathscr{A}_{\alpha}^{p}\right)^{*}=\left(\mathscr{A}_{\tau}^{1}\right)^{*}$, and so

$$
\left|C_{\varphi} f(z)\right| \leq c(\tau, 1)\left(\frac{1}{1-|\varphi(z)|^{2}}\right)^{2+\tau}=c(\tau, 1)\left(\frac{1}{1-|\varphi(z)|^{2}}\right)^{(2+\alpha) / p}
$$

even for all $f \in B_{\mathscr{A}_{r}}$.

We obtain the following consequences:

COROLlaRY 7. Let $\alpha, \alpha^{\prime}, \beta \geq-1$ and $\varphi \in \Phi$ be given and suppose that $0<$ $p, p^{\prime}, q, q^{\prime}, s<\infty$ are such that $q, q^{\prime}, s q \geq 1$.

(a) If $C_{\varphi}$ is order bounded as an operator $\mathscr{A}_{\alpha}^{p} \rightarrow \mathscr{A}_{\beta}^{q}$, then it is order bounded as an operator $\mathscr{A}_{\alpha}^{\text {ps }} \rightarrow \mathscr{A}_{\alpha}^{q s}$, too. 
(b) If $(2+\alpha) p^{\prime} q=\left(2+\alpha^{\prime}\right) p q^{\prime}$, then $C_{\varphi}$ is order bounded as an operator $\mathscr{A}_{\alpha}^{p} \rightarrow \mathscr{A}_{\beta}^{q}$ if and only if it exists as an order bounded operator $\mathscr{A}_{\alpha^{\prime}}^{p^{\prime}} \rightarrow \mathscr{A}_{\beta}^{q^{\prime}}$.

We know from Corollary 5 that in case $q \geq p$ and with $\alpha^{\prime}=(2+\alpha)(q / p)-2$, $C_{\varphi}$ acts as a Hilbert space operator $\mathscr{A}_{\alpha^{\prime}}^{2} \rightarrow \mathscr{A}_{\beta}^{2}$. By (b), it is order bounded provided we start with an order bounded operator $\mathscr{A}_{\alpha}^{p} \rightarrow \mathscr{A}_{\beta}^{q}$. But for an operator $u: H \rightarrow$ $K$ between Hilbert spaces, order boundedness is equivalent to $u$ being a HilbertSchmidt operator: $\sum_{i \in I}\left\|u e_{i}\right\|^{2}$ converges for some, and then every, orthonormal basis $\left(e_{i}\right)_{i \in I}$ of $H$; compare with [3, Chapters 4-5]. If $\alpha^{\prime}>-1$, then the functions $z \mapsto \mathrm{B}\left(\alpha^{\prime}+1, n+1\right)^{-1 / 2} z^{n}, n=0,1,2, \ldots$ form an orthonormal basis in $\mathscr{A}_{\alpha^{\prime}}^{2}$; here $\mathrm{B}\left(\alpha^{\prime}+1, n+1\right)=\Gamma\left(\alpha^{\prime}+1\right) \Gamma(n+1) \Gamma\left(\alpha^{\prime}+n+2\right)^{-1}$ is the Beta function. $\alpha^{\prime}=-1$ can only occur when $\alpha=-1$ and $p=q$; the functions $z \mapsto z^{n}$ then form an orthonormal basis in $\mathscr{A}_{\alpha^{\prime}}^{2}=H^{2}$. In any case, we get:

Corollary 8. Let $\alpha, \beta \geq-1,0<p \leq q<\infty$ and $\varphi \in \Phi$ be given, and let $\alpha^{\prime}=(2+\alpha)(q / p)-2$. The following are equivalent statements:

(i) $C_{\varphi}$ exists as an order bounded operator from $\mathscr{A}_{\alpha}^{p}$ to $\mathscr{A}_{\beta}^{q}$,

(ii) $C_{\varphi}$ acts as a Hilbert-Schmidt operator $\mathscr{A}_{\alpha^{\prime}}^{2} \rightarrow \mathscr{A}_{\beta}^{2}$,

(iii) $\sum_{n=0}^{\infty} c_{n}^{2}\left\|\varphi^{n}\right\|_{\beta .2}^{2}<\infty$, where $c_{n}=\mathrm{B}\left(\alpha^{\prime}+1, n+1\right)^{-1 / 2}$ if $\alpha^{\prime}>-1$ and $c_{n}=1$ otherwise.

(iv) $\left(\left\|\varphi^{n}\right\|_{\beta, q}\right)_{n}$ belongs to the Lorentz sequence space $\ell^{p /(2+\alpha) \cdot q}$.

The incorporation of (iv) is by a standard binomial series argument and amounts to requiring convergence of the series $\sum_{n=0}^{\infty}(n+1)^{(2+\alpha)(q / p)-1}\left\|\varphi^{n}\right\|_{\beta, q}^{q}$. Compare also with [5], and with [7] for the special case of Hardy spaces.

\section{Absolutely summing operators}

Order bounded operators are close to $p$-integral and to (absolutely) $p$-summing operators; see [12] and [3, Chapter 5]. In fact, if $v$ is any measure, if $Y$ is a subspace of $L^{q}(v)$, and if $u: X \rightarrow Y$ is order bounded, then it is $q$-summing (and even $q$-integral), and if $u^{*}: Y^{*} \rightarrow X^{*}$ is $q$-summing, then $u$ is order bounded.

Proposition 6 does not carry over to summing operators. For example if $p \geq 1$ and $2+\alpha>p$, then Grothendieck's inequality yields that $C_{\varphi}: \mathscr{A}_{\alpha}^{(p)} \rightarrow \mathscr{A}_{\beta}^{2}$ is 1 -summing whenever $C_{\varphi}$ exists as a bounded operator $\mathscr{A}_{\alpha}^{p} \rightarrow \mathscr{A}_{\beta}^{2}$ (since $\mathscr{A}_{\alpha}^{(p)}$ is isomorphic to $\left.\ell^{\mathrm{j}}\right)$.

The situation is different for operators whose adjoints have the summing properties under consideration. We limit ourselves to the case of 'genuine' Bergman spaces. Results for Hardy spaces are already contained in [4, 5, 8] and [16]. 
PROPOSITION 9. Let $\varphi \in \Phi$ be given and suppose that $1<p, p^{\prime}, q, q^{\prime}<\infty$ and $\alpha, \beta>-1$ are such that $p \leq q \leq p^{*}, p^{\prime} \leq q^{\prime} \leq p^{\prime *}$ and $(2+\alpha) p^{\prime} q=\left(2+\alpha^{\prime}\right) p q^{\prime}$. Then $C_{\varphi}: \mathscr{A}_{\alpha}^{p} \rightarrow \mathscr{A}_{\beta}^{q}$ exists as an operator with a $q$-summing adjoint if and only if $C_{\varphi}: \mathscr{A}_{\alpha^{\prime}}^{p^{\prime}} \rightarrow \mathscr{A}_{\beta}^{q^{\prime}}$ exists and has a $q^{\prime}$-summing adjoint.

PROOF. Suppose that $C_{\varphi}$ maps $\mathscr{A}_{\alpha^{\prime}}^{p^{\prime}}$ into $\mathscr{A}_{\beta}^{q^{\prime}}$ and has $q^{\prime}$-summing adjoint. By the ideal properties of such operators, $C_{\varphi}: \mathscr{A}_{\alpha^{\prime}}^{\left(p^{\prime}\right)} \rightarrow \mathscr{A}_{\beta}^{q^{\prime}}$ also has a $q^{\prime}$-summing adjoint and is hence order bounded. By Proposition 6, $C_{\varphi}: \mathscr{A}_{\alpha}^{p} \rightarrow \mathscr{A}_{\beta}^{q}$ is order bounded. We write $\varrho:=(2+\alpha) / p-(1 / q)$ and conclude that $\sum_{n=0}^{\infty}(n+1)^{\rho q}\left\|\varphi^{n}\right\|_{\beta, q}^{q}$ is finite. Therefore $u: \ell^{q^{*}} \rightarrow \mathscr{A}_{\beta}^{q}: \xi=\left(\xi_{n}\right) \mapsto \sum_{n=0}^{\infty}(n+1)^{e} \xi_{n} \varphi^{n}$ is well defined and bounded. Note that $u^{*}$ is $q$-nuclear and so in particular $q$-summing (see [3, 12]).

We claim that $f \mapsto\left((n+1)^{-} \widehat{\widehat{f}}(n)\right)_{n \in \mathbb{N}_{0}}$ defines a bounded operator $v: \mathscr{A}_{\alpha}^{p} \rightarrow \ell^{q^{*}}$; as usual, we write here $f=\sum_{n=0}^{\infty} \widehat{f}(n) z^{n}(z \in D)$. From the obvious relation $C_{\varphi}=u v$ we can then conclude that $C_{\varphi}$, together with $u$, has a $q$-summing adjoint.

To this end we set $\delta:=1+\alpha-Q$, so that $\delta=(2+\alpha) / p^{*}-\left(1 / q^{*}\right)$. We will prove that $\xi=\left(\xi_{n}\right)_{n} \mapsto \sum_{n=0}^{\infty} \xi_{n}(n+1)^{\delta} z^{n}$ defines a bounded operator $w: \ell^{q} \rightarrow \mathscr{A}_{\alpha}^{p^{*}}$; here $z^{n}$ is used to denote the $n$-th monomial $z \mapsto z^{n}$. We employ a result of Sledd [17] according to which, for some constant $c_{1}$ and every $\xi \in \ell^{q}$,

$$
\|w(\xi)\|_{\mathscr{\alpha}_{\alpha}^{p^{*}}}^{p^{*}} \leq c_{1}\left(\left|\xi_{0}\right|+\sum_{n=0}^{\infty} 2^{-n(1+\alpha)}\left\|\sum_{k=2^{n}}^{2^{n+1}-1} \xi_{k}(k+1)^{\delta} z^{k}\right\|_{H^{p^{*}}}^{p^{*}}\right) ;
$$

the two expressions are even comparable to each other. Since $p^{*} \geq 2$, the HausdorffYoung inequality provides access to the right hand side: for each $n$, we have

$$
\left\|\sum_{k=2^{n}}^{2^{n+1}-1} \xi_{k}(k+1)^{\delta} z^{k}\right\|_{H^{p^{*}}}^{p^{*}} \leq\left(\sum_{k=2^{n}}^{2^{n+1}-1}\left|\xi_{k}\right|^{p}(k+1)^{\delta p}\right)^{p^{*} / p} .
$$

This is further estimated using Hölder's inequality [with $q / p$ and $q /(q-p)$ ]

$$
\begin{aligned}
\left\|\sum_{k=2^{n}}^{2^{n+1}-1} \xi_{k}(k+1)^{\delta} z^{k}\right\|_{H^{p^{*}}}^{p^{*}} & \leq\left(\sum_{k=2^{n}}^{2^{n+1}-1}\left|\xi_{k}\right|^{q}\right)^{p^{*} / q}\left(\sum_{k=2^{n}}^{2^{n+1}-1}(k+1)^{\delta} \frac{p q}{q-p}\right)^{p^{*}(q-p) /(p q)} \\
& \leq c_{2}\left(\sum_{k=2^{n}}^{2^{n+1}-1}\left|\xi_{k}\right|^{q}\right)^{p^{*} / q} 2^{n\left(\delta p^{*}+p^{*}(q-p) /(p q)\right)} \\
& =c_{2}\left(\sum_{k=2^{n}}^{2^{n+1}-1}\left|\xi_{k}\right|^{q}\right)^{p^{*} / q} 2^{n(1+\alpha)}
\end{aligned}
$$


$c_{2}$ being a certain constant. Since $p^{*} \geq q$ it follows that there is a further constant $c$ such that

$$
\|w(\xi)\|_{\mathscr{A}_{a}^{p^{*}}}^{p^{*}} \leq c \sum_{n=0}^{\infty}\left(\sum_{k=2^{n}}^{2^{n+1}-1}\left|\xi_{k}\right|^{q}\right)^{p^{*} / q} \leq c\|\xi\|_{q}^{p^{*}} .
$$

It is well known that $\langle f, g\rangle_{\alpha}:=\int_{D} f \bar{g} d \sigma_{\alpha}$ puts $\mathscr{A}_{\alpha}^{p}$ and $\mathscr{A}_{\alpha}^{p^{*}}$ in duality and establishes an isomorphism between $\mathscr{A}_{\alpha}^{p^{*}}$ and $\left(\mathscr{A}_{\alpha}^{p}\right)^{*} ;$ moreover, $\left|\left\langle f, z^{n}\right\rangle_{\alpha}\right|$ and $|\widehat{f}(n)|(n+1)^{-1-\alpha}$ are comparable for all $f \in \mathscr{A}_{\alpha}^{p}$ and all $n \in \mathbb{N}_{0}$. This implies that there is a constant $C$ such that $\|v f\|_{\ell^{*}} \leq C\left\|w^{*} f\right\|_{\ell^{*}}\left(f \in \mathscr{A}_{\alpha}^{p}\right)$, which was what we wanted.

Again, the property under discussion can entirely be reformulated in terms of Hilbert spaces:

COROLlaRY 10. Let $\varphi \in \Phi, \alpha, \beta>-1$ and $1<p \leq q \leq p^{*}$ be given. Set $\alpha^{\prime}=(2+\alpha)(q / p)-2$. Then $C_{\varphi}: \mathscr{A}_{\alpha}^{p} \rightarrow \mathscr{A}_{\beta}^{q}$ exists and has a $q$-summing adjoint if and only if $C_{\varphi}: \mathscr{A}_{\alpha^{\prime}}^{2} \rightarrow \mathscr{A}_{\beta}^{2}$ exists and is a Hilbert-Schmidt operator.

This is immediate from Proposition 9 since a Hilbert space operator is HilbertSchmidt if and only if it is $r$-summing for some (and then all) $r$ (see [3, 12]).

\section{References}

[1] C. Cowen-B. MacCluer, Composition operators on spaces of analytic functions (CRC Press, Boca Raton, 1995).

[2] J. Diestel, Sequences and series in Banach spaces (Springer, New York, 1984).

[3] J. Diestel, H. Jarchow and A. Tonge, Absolutely summing operators (Cambridge University Press, Cambridge, 1995).

[4] T. Domenig, Composition operators on weighted Bergman spaces and Hardy spaces (Dissertation, Universität Zürich, 1997).

[5] — _ 'Order bounded and $p$-summing composition operators', Contemp. Math. 213 (1998), $27-41$.

[6] P. Duren, Theory of $H^{p}$ spaces (Academic Press, New York, 1970).

[7] H. Hunziker and H. Jarchow, 'Composition operators which improve integrability', Math. Nachr. 122 (1991), 83-99.

[8] H. Jarchow, 'Absolutely summing composition operators', in: Proc. Conf. Funct. Anal. (Marcel Dekker, 1993) pp. 193-203.

[9] _ - 'Compactness properties of composition operators', submitted.

[10] H. Jarchow and R. Riedl, 'Factorization of composition operators through Bloch type spaces', Illinois J. Math. 39 (1995), 431-440.

[11] D. H. Luecking, 'Representation and duality in weighted spaces of analytic functions', Indiana Univ. Math. J. 34 (1985), 319-336.

[12] A. Pietsch, Operator ideals (North-Holland, Amsterdam, 1980). 
[13] R. Riedl, Composition operators and geometric properties of analytic functions (Dissertation, Universität Zürich, 1994).

[14] J. H. Shapiro, 'Mackey topologies, reproducing kernels, and diagonal maps on the Hardy and Bergman spaces', Duke Math. J. 43 (1976), 187-202.

[15] - Composition operators and classical function theory (Springer, New York, 1993).

[16] J. H. Shapiro and P. D. Taylor, 'Compact, nuclear, and Hilbert-Schmidt composition operators on $H^{2}$, Indiana Univ. Math. J. 23 (1973), 471-496.

[17] W. T. Sledd, 'Some results about spaces of analytic functions introduced by Hardy and Littlewood', Michigan Math. J. 31 (1984), 307-319.

[18] W. Smith, 'Composition operators between Bergman and Hardy spaces', Trans. Amer. Math. Soc. 348 (1996), 2331-2348.

[19] K. Zhu, Operator theory in function spaces (Marcel Dekker, New York, 1990).

Institut für Mathematik

Universität Zürich

Winterthurerstr. 190

CH 8057 Zürich

Switzerland

e-mail: domenig@amath.unizh.ch, jarchow@math.unizh.ch, riedl@ifi.unizh.ch 